\title{
About periodic and quasi-periodic orbits of a new type for twist maps of the torus
}

\author{
SALVADOR ADDAS-ZANATA \\ Department of Mathematics, Princeton University \\ Fine Hall-Washington Road, Princeton, NJ 08544-1000, USA \\ Manuscript received on August 08, 2001; accepted for publication on November 26, 2001; \\ presented by J. SOTOMAYOR
}

\begin{abstract}
We prove that for a large and important class of $C^{1}$ twist maps of the torus periodic and quasiperiodic orbits of a new type exist, provided that there are no rotational invariant circles (R.I.C's). These orbits have a non-zero "vertical rotation number" (V.R.N.), in contrast to what happens to Birkhoff periodic orbits and Aubry-Mather sets. The V.R.N. is rational for a periodic orbit and irrational for a quasi-periodic. We also prove that the existence of an orbit with a $V \cdot R \cdot N=a>0$, implies the existence of orbits with $V \cdot R \cdot N=b$, for all $0<b<a$. And as a consequence of the previous results we get that a twist map of the torus with no R.I.C's has positive topological entropy, which is a very classical result. In the end of the paper we present some applications and examples, like the Standard map, such that our results apply.
\end{abstract}

Key words: twist maps, rotational invariant circles, topological methods, vertical rotation number, Nielsen-Thurston theory.

\section{INTRODUCTION}

In this announcement we describe results on the existence and on the structure of some periodic and quasi-periodic orbits of a new type for twist maps of the torus. These orbits exist, if and only if, the twist map does not have rotational invariant circles (R.I.C), and in some important cases they are related to escaping and transport problems.

Given a one parameter family $T_{\mu}$ of twist maps of the torus, such that for $\mu>\mu_{0}$ there are no R.I.C's, as a consequence of the break up of the last invariant circle we have, besides many important results that have already been studied by several authors, the appearance of these new type of orbits.

E-mail: szanata@math.princeton.edu 
For us, twist maps are $C^{1}$ diffeomorphisms of the cylinder (or annulus, torus) onto itself that have the following property: The angular component of the image of a point increases as the radial component of the point increases (more precise definitions will be given below). Such maps were first studied in connection with the three-body problem by Poincare and later it was found that first return maps for many problems in Hamiltonian dynamics are actually twist maps. Although they have been extensively studied, there are still many open questions about their dynamics. A great progress has been achieved in the nearly integrable case, by means of KAM theory, see (Moser 1973), and many important results have been proved in the general case, concerning the existence of periodic and quasi-periodic orbits, the Aubry-Mather sets, see (Mather 1982) and (Aubry and LeDaeron 1983).

Before presenting the results, we need some notations and definitions.

\section{NOTATION AND DESCRIPTIONS}

$0)$ Let $(\phi, I)$ denote the coordinates for the cylinder $S^{1} \times \mathbb{R}=\mathbb{R} / \mathbb{Z} \times \mathbb{R}$, where $\phi$ is defined modulo 1. Let $(\widetilde{\phi}, \widetilde{I})$ denote the coordinates for the universal cover, $\mathbb{R}^{2}$. For all maps $\widehat{f}$ : $S^{1} \times \mathbb{R} \rightarrow S^{1} \times \mathbb{R}$ we define $\left(\phi^{\prime}, I^{\prime}\right)=\widehat{f}(\phi, I)$ and $\left(\widetilde{\phi}^{\prime}, \widetilde{I}^{\prime}\right)=f(\widetilde{\phi}, \widetilde{I})$, where $f: \mathbb{R}^{2} \rightarrow \mathbb{R}^{2}$ is a lift of $\widehat{f}$.

1) $D_{r}^{1}\left(\mathbb{R}^{2}\right)=\left\{f: \mathbb{R}^{2} \rightarrow \mathbb{R}^{2} / f\right.$ is a $C^{1}$ diffeomorphism of the plane, $\widetilde{I}^{\prime}(\widetilde{\phi}, \widetilde{I}) \stackrel{\widetilde{I} \rightarrow \pm \infty}{\rightarrow} \pm \infty$, $\partial_{\tilde{I}} \widetilde{\phi}^{\prime}>0$ (twist to the right), $\widetilde{\phi}^{\prime}(\widetilde{\phi}, \widetilde{I}) \stackrel{\widetilde{I} \rightarrow \pm \infty}{\rightarrow} \pm \infty$ and $f$ is the lift of a $C^{1}$ diffeomorphism $\left.\widehat{f}: S^{1} \times \mathbb{R} \rightarrow S^{1} \times \mathbb{R}\right\}$.

2) $\operatorname{Diff}_{r}^{1}\left(S^{1} \times \mathbb{R}\right)=\left\{\widehat{f}: S^{1} \times \mathbb{R} \rightarrow S^{1} \times \mathbb{R} / \widehat{f}\right.$ is induced by an element of $\left.D_{r}^{1}\left(\mathbb{R}^{2}\right)\right\}$.

3) Let $p_{1}: \mathbb{R}^{2} \rightarrow \mathbb{R}$ and $p_{2}: \mathbb{R}^{2} \rightarrow \mathbb{R}$ be the standard projections, respectively in the $\widetilde{\phi}$ and $\widetilde{I}$ coordinates $\left(p_{1}(\widetilde{\phi}, \widetilde{I})=\widetilde{\phi}\right.$ and $\left.p_{2}(\widetilde{\phi}, \widetilde{I})=\widetilde{I}\right)$. We also use $p_{1}$ and $p_{2}$ for the standard projections of the cylinder.

4) Given a measure $\mu$ on the cylinder that is positive on open sets and a map $\widehat{T} \in \operatorname{Diff}_{r}^{1}\left(S^{1} \times \mathbb{R}\right)$ we say that $\widehat{T}$ is $\mu$-exact if $\mu$ is invariant under $\widehat{T}$ and for any open set $A$ homeomorphic to the cylinder we have:

$$
\mu(\widehat{T}(A) \backslash A)=\mu(A \backslash \widehat{T}(A))
$$

5) Let $T Q \subset D_{r}^{1}\left(\mathbb{R}^{2}\right)$ be such that for all $T \in T Q$ we have:

$$
\begin{gathered}
T:\left\{\begin{array}{l}
\widetilde{\phi}^{\prime}=T_{\phi}(\widetilde{\phi}, \widetilde{I}) \\
\widetilde{I}^{\prime}=T_{I}(\widetilde{\phi}, \widetilde{I})
\end{array}\right. \text { and: } \\
\left\{\begin{array}{c}
T_{I}(\widetilde{\phi}+1, \widetilde{I})=T_{I}(\widetilde{\phi}, \widetilde{I}) \\
T_{I}(\widetilde{\phi}, \widetilde{I}+1)=T_{I}(\widetilde{\phi}, \widetilde{I})+1 \\
T_{\phi}(\widetilde{\phi}+1, \widetilde{I})=T_{\phi}(\widetilde{\phi}, \widetilde{I})+1 \\
T_{\phi}(\widetilde{\phi}, \widetilde{I}+1)=T_{\phi}(\widetilde{\phi}, \widetilde{I})+1
\end{array}\right.
\end{gathered}
$$


Every $T \in T Q$ induces a map $\widehat{T} \in \operatorname{Diff}_{r}^{1}\left(S^{1} \times \mathbb{R}\right)$ and a map $\bar{T}: T^{2} \rightarrow T^{2}$, where $T^{2}=\mathbb{R}^{2} / \mathbb{Z}^{2}$ is the 2-torus and $p: \mathbb{R}^{2} \rightarrow T^{2}$ is the associated covering map.

6) Let $T \in T Q$ :

- We say that a set $O_{x}=\left\{x, \bar{T}(x), \ldots, \bar{T}^{n-1}(x)\right\} \subset T^{2}$ is a (vertical) $n$-periodic orbit (or set), if $\bar{T}^{n}(x)=x, \bar{T}^{i}(x) \neq \bar{T}^{j}(x)$, for $i \neq j$, with $i, j \in\{0,1, \ldots, n-1\}$ and

$$
\rho_{V}(x)=\lim _{n \rightarrow \infty} \frac{p_{2} \circ T^{n}(\tilde{x})-p_{2}(\tilde{x})}{n} \in \mathbb{Q}^{*}
$$

for any $\tilde{x} \in p^{-1}(x)$. It is clear from the definition of $T Q$ that $\rho_{V}(x)$ is the same for all $\tilde{x} \in p^{-1}(x)$, so it is well defined.

- We say that a set $Q$ is a (vertical) quasi-periodic set for $\bar{T}$ if $Q$ is a compact $\bar{T}$-invariant set and the number

$$
\rho_{V}(x)=\lim _{n \rightarrow \infty} \frac{p_{2} \circ T^{n}(\tilde{x})-p_{2}(\tilde{x})}{n} \in \mathbb{R} \backslash \mathbb{Q}
$$

exists and is the same for all $x \in Q$.

It can be proved that when $\rho_{V}(x) \neq 0$, we have

$$
\lim _{n \rightarrow \infty}\left|\frac{p_{1} \circ T^{n}(\tilde{x})-p_{1}(\tilde{x})}{n}\right|=\infty, \text { for all } \tilde{x} \in p^{-1}(x),
$$

so in a certain way these orbits are completely characterized by the vertical rotation number $\rho_{V}$.

Before presenting the main results we need another definition:

- Given a map $T \in T Q$ such that $\widehat{T}$ is $\mu$-exact we say that $C$ is a rotational invariant circle (R.I.C.) for $T$ if $C$ is a homotopically non-trivial simple closed curve on the cylinder and $\widehat{T}(C)=C$. By a theorem essentially due to Birkhoff, $C$ is the graph of some Lipschitz function $\psi: S^{1} \rightarrow \mathbb{R}$, see (Katok and Hasselblatt 1995), page 430.

The following theorems are the main results of this announcement:

Theorem 1. Let $T \in T Q$ be such that $\widehat{T}$ is $\mu$-exact. Then:

- given $k \in \mathbb{Z}^{*}, \exists N>0$, such that $\bar{T}$ has a periodic orbit with $\rho_{V}$ (vertical rotation number) $=\frac{k}{N}$, if and only if, $T$ does not have R.I.C's.

The next theorem shows how these periodic orbits appear:

THEOREM 2. Again, for all $T \in T Q$ such that $\widehat{T}$ is $\mu$-exact, if $\bar{T}$ has a periodic orbit with $\rho_{V}=\frac{k}{N}$, then for every pair $\left(k^{\prime}, N^{\prime}\right) \in \mathbb{Z}^{*} \times \mathbb{N}^{*}$, such that $0<\left|\frac{k^{\prime}}{N^{\prime}}\right|<\left|\frac{k}{N}\right|$ and $k . k^{\prime}>0, \bar{T}$ has a periodic orbit with rotation number $\rho_{V}^{\prime}=\frac{k^{\prime}}{N^{\prime}}$.

About the quasi-periodic orbits we have the following:

THEOREM 3. For all $T \in T Q$ such that $\widehat{T}$ is $\mu$-exact we have:

If $\bar{T}$ has an orbit with $\rho_{V}=\omega$ (periodic if $\omega \in \mathbb{Q}$ and quasi-periodic if not), then for all $\omega^{\prime} \in \mathbb{R} \backslash \mathbb{Q}$ 
such that $0<\left|\omega^{\prime}\right|<|\omega|$ and $\omega . \omega^{\prime}>0, \bar{T}$ has a quasi-periodic set with vertical rotation number $\rho_{V}^{\prime}=\omega^{\prime}$.

As a consequence of the proof of Theorem (3) we get a new proof for the following classical result:

THEOREM 4. Every $T \in T Q$ without R.I.C's such that $\widehat{T}$ is $\mu$-exact induces a map $\bar{T}: T^{2} \rightarrow T^{2}$ such that $h(\bar{T})>0$, where $h(\bar{T})$ is the topological entropy of $\bar{T}$.

Theorems (1), (2) and (3) are proved using topological ideas, essentially due to the twist condition and some results due to (Le Calvez 1986,1991). In the proofs of theorems (3) and (4) we also use some results from the Nielsen-Thurston theory of classification of homeomorphisms of surfaces up to isotopy, to isotope the map to a pseudo-Anosov one, see (Llibre and Mackay 1991) and (Thurston 1988), and then some results due to (Handel 1990), to prove the existence of quasi-periodic orbits with irrational vertical rotation number.

\section{EXAMPLES AND APPLICATIONS}

1) It is obvious that the well-known Standard map $S_{M}: T^{2} \rightarrow T^{2}$ given by

$$
S_{M}:\left\{\begin{array}{l}
\phi^{\prime}=\phi+I^{\prime}(\bmod 1) \\
I^{\prime}=I-\frac{k}{2 \pi} \sin (2 \pi \phi)(\bmod 1)
\end{array} \text { is induced by an element of } T Q .\right.
$$

Also, it is easy to see that its generating function is:

$$
h_{S_{M}}\left(\phi, \phi^{\prime}\right)=\frac{\left(\phi^{\prime}-\phi\right)^{2}}{2}+\frac{k}{4 \pi^{2}} \cdot \cos (2 \pi \phi) \Rightarrow h_{S_{M}}\left(\phi+1, \phi^{\prime}+1\right)=h_{S_{M}}\left(\phi, \phi^{\prime}\right),
$$

so $S_{M}$ is a Lebesgue-exact map. In this way, as we know that for sufficiently large $k>0$ $S_{M}$ does not have R.I.C's, we can apply our previous results to this family of maps. In fact, theorems (1) and (2) can be used to produce a new criteria to obtain estimates for the parameter value $k_{c r}$, which is defined in the following way: if $k>k_{c r}$, then $S_{M}$ does not have R.I.C and for $k \leq k_{c r}$ there is at least one R.I.C. This happens because for each $1 / n, n \in \mathbb{N}^{*}$, we can calculate a number $k_{n}$, such that for $k \geq k_{n}, S_{M}$ has a $n$-periodic orbit with $\rho_{V}=1 / n$ and for $k<k_{n}$ it does not have such an orbit. From the theorems cited above, if $n>m$ then $k_{n} \leq k_{m}$ and $\lim _{n \rightarrow \infty} k_{n}=k_{c r}$.

2) In (Addas-Zanata 2000) the dynamics near a homoclinic loop to a saddle-center equilibrium of a 2-degrees of freedom Hamiltonian system was studied by means of an approximation of a certain Poicaré map. In an appropriate coordinate system this map is given by:

$$
\widehat{F}: S^{1} \times \mathbb{R} \hookleftarrow:\left\{\begin{array}{l}
\phi^{\prime}=F_{\phi}(\phi, I)=m(\phi)+I^{\prime}(\bmod \pi) \\
I^{\prime}=F_{I}(\phi, I)=\gamma \log (J(\phi))+I
\end{array}\right.
$$

where

$$
\begin{aligned}
& J(\phi)=\alpha^{2} \cos ^{2}(\phi)+\alpha^{-2} \sin ^{2}(\phi), J(\phi+\pi)=J(\phi) \\
& m(\phi)=\arctan \left(\frac{\tan (\phi)}{\alpha^{2}}\right), m(0)=0, m(\phi+\pi)=m(\phi)+\pi
\end{aligned}
$$


A simple calculation shows that in these coordinates $\widehat{F}$ is $\mu$-exact and $\mu$ is given by:

$$
\mu(A)=\int_{A} e^{\frac{I}{\gamma}} d \phi d I
$$

It is also easy to see that $\widehat{F}$ induces a map $\bar{F}: T^{2} \rightarrow T^{2}\left(T^{2}=\mathbb{R}^{2} /(\pi \mathbb{Z})^{2}\right)$ just by taking $I^{\prime}=F_{I}(\phi, I)(\bmod \pi)$ in expression (3) and this torus map is also induced by an element of $T Q$.

And from (Grotta Ragazzo 1997), $\exists \alpha_{\text {crit }}(\gamma)$ such that for $\alpha>\alpha_{\text {crit }}(\gamma), \bar{F}$ does not have R.I.C's. In this case, we can apply the same criteria explained for the standard map. But as there are 2 parameters, we do not obtain a critical value, we obtain a critical set in the $(\gamma, \alpha)$ plane. Another important application of this theory is to obtain properties about the structure of the unstable set of the above mentioned homoclinic loop (to the saddle-center equilibrium), when the former is unstable. The periodic orbits given by theorem (1) were analyzed in (Addas-Zanata and Grotta Ragazzo 2001) and it was proved that for every vertical rotation number $\frac{m}{n}>0$, there is an open set in the parameter space with a $\frac{m}{n}$-periodic orbit which is topologically a sink. In particular, it can be proved that, for a fixed value of $\gamma>0$, given an $\epsilon>\alpha_{c r}(\gamma)>1$, where $\alpha_{c r}(\gamma)$ is analogous to the constant $k_{c r}$ defined for the standard map, there is a number $\frac{m}{n}>0$ and an open interval $I \frac{m}{n} \subset\left(\alpha_{c r}(\gamma), \epsilon\right)$, such that for $\alpha \in I \frac{m}{n}, \bar{F}$ has a vertical periodic orbit with $\rho_{V}=\frac{m}{n}$ which is also a topological sink. So we can say that one of the mechanisms that cause the lost of stability of the homoclinic loop is the creation of periodic sinks for $\bar{F}$. And in (Addas-Zanata and Grotta Ragazzo 2000) it was proved that the existence of a topological sink for $\bar{F}$ implies many interesting properties on the topology of the set of orbits that have the saddle-center loop as their $\alpha$-limit set (a set analogous to the unstable manifold of a hyperbolic periodic orbit). More precisely, in this case, given an arbitrary neighborhood of the original homoclinic loop, a set of positive measure contained in this neighborhood escapes from it following (or clustering around) a finite set of orbits that in a certain sense, correspond to the topological sinks for $\bar{F}$. In a forthcoming paper, we will analyze the following function:

$$
\rho_{V}^{\max }(\gamma, \alpha)=\sup _{P \in T^{2}} \rho_{V}(P)=\sup _{P \in T^{2}}\left[\lim _{n \rightarrow \infty} \frac{p_{2} \circ F^{n}(P)-p_{2}(P)}{n}\right],
$$

where the supremum is taken over all $P \in T^{2}$ such that $\rho_{V}(P)$ exists. Using a method developed in (Addas-Zanata and Grotta Ragazzo 2001), we plan to prove the density of periodic sinks in the subset of the parameter space $(\gamma, \alpha)$ where $\bar{F}$ does not have R.I.C's.

\section{ACKNOWLEDGMENTS}

I am very grateful to C. Grotta Ragazzo for listening to oral expositions of these results, reading the first manuscripts and for many discussions, comments and all his support.

Supported by CNPq, grant number: 200564/00-5 (part of this work was done while the author was under support by FAPESP, grant number: 96/08981-3). 


\section{RESUMO}

Provamos que para uma relevante classe de aplicações $C^{1}$ no toro, que desviam a vertical para a direita, existem órbitas periódicas e quase-periódicas de um novo tipo, se e somente se, não existem círculos rotacionais invariantes. Essas órbitas têm um número de rotação vertical não nulo (N.R.V), em contraste com o que ocorre para órbitas periódicas do tipo Birkhoff e para os conjuntos de Aubry-Mather. O número de rotação vertical é racional para uma órbita periódica e irracional para uma quase-periódica. Também provamos que a existência de uma órbita com $N . R . V=a$ implica a existência de órbitas com N.R.V $=b$, para todo $0<b<a$. Como consequência destes resultados, obtemos que uma aplicação do toro que desvia a vertical e não possui círculos rotacionais invariates, necessariamente tem entropia topológica positiva, que é um resultado clássico. No fim deste trabalho apresentamos aplicações e exemplos, como o Standard map, dos resultados obtidos.

Palavras-chave: aplicações que desviam a vertical, círculos rotacionais invariantes, métodos topológicos, número de rotação vertical, teoria de Nielsen-Thurston.

\section{REFERENCES}

Addas-Zanata S. 2000. On the dynamics of twist maps of the torus. Doctoral Thesis. In Portuguese. IMEUSP, Brasil, 123p.

Addas-Zanata S and Grotta Ragazzo C. 2000. Conservative dynamics: unstable sets for saddle-center loops. submitted.

Addas-Zanata S and Grotta Ragazzo C. 2001. On the stability of some periodic orbits of a new type for twist maps. Submitted.

Aubry S and Le Daeron P. 1983. The discrete Frenkel-Kontorova model and its extensions. Physica D 8: $381-422$.

Grotta Ragazzo C. 1997. On the Stability of Double Homoclinic Loops. Comm Math Phys 184: 251-272.

Handel M. 1990. The Rotation Set of a Homeomorphism of the Annulus is Closed. Comm Math Phys 127: 339-349.

Katok A and Hasselblatt B. 1995. Introduction to Modern Theory of Dynamical Systems. Cambridge, Cambridge University Press, 802p.

Le Calvez P. 1986. Existence d'orbites quasi-périodiques dans les attracteurs de Birkhoff. Comm Math Phys 106: 383-394.

Le Calvez P. 1991. Propriétés Dynamiques des Difféomorphismes de L'Anneau et du Tore. Astérisque 204, 160p.

Llibre J AND MACKAY R. 1991. Rotation vectors and entropy for homeomorphisms of the torus isotopic to the identity. Ergod Th and Dynam Sys 11: 115-128.

Mather J. 1982. Existence of quasi-periodic orbits for twist homeomorphisms of the annulus. Topology. 21(4): 457-467. 
Moser J. 1973. Stable and Random Motions in Dynamical Systems. Princeton, Princeton University Press, $170 \mathrm{p}$.

Thurston W. 1988. On the Geometry and Dynamics of Diffeomorphisms of Surfaces. Bull Amer Math Society 19(2): 417-431. 